\title{
Discovering the Prevalence of Antimicrobial Resistance Through Creation of an Antibiogram in a Rural Kenyan Hospital
}

lan C Drobish

University of San Diego

George Otieno

AIC Kijabe Hospital

Janet Mihang'o

AIC Kijabe Hospital

Virginia D Barnette

AIC Kijabe Hospital

Grace Njoroge Waceke

AIC Kijabe Hospital

Solomon Thuo

AIC Kijabe Hospital

Kaya S Belknap

AIC Kijabe Hospital

Katy Linley

AIC Kijabe Hospital

Arianna McLain Shirk

AIC Kijabe Hospital

S Taylor McClanahan

AIC Kijabe Hospital

Elizabeth Irungu

AIC Kijabe Hospital

Faith Lelei

AlC Kijabe Hospital

Immaculate K Barasa

AIC Kijabe Hospital

B Jason Brotherton ( $\sim$ jb042107@gmail.com )

AlC Kijabe Hospital https://orcid.org/0000-0003-1144-6107 
Keywords: antimicrobial resistance, multidrug resistance, antimicrobial stewardship, LMIC, global health, Kenya, infectious disease, sub-Saharan Africa

Posted Date: January 31st, 2022

DOI: https://doi.org/10.21203/rs.3.rs-1224260/v1

License: (c) (1) This work is licensed under a Creative Commons Attribution 4.0 International License. Read Full License 


\section{Abstract}

\section{Background}

Antimicrobial resistance is a pressing global health issue. Data are lacking in detailing the presence and burden of antimicrobial resistance in low and middle-income countries. What is currently available is quarantined to large, urban centers away from the rural facilities.

\section{Methods}

This was a retrospective descriptive study performed at Kijabe Hospital, a rural 350-bed teaching hospital, from February 2016 to September 2020. Cultures from blood, urine, and cerebrospinal fluid were included from all pediatric and adult patients. Data was analyzed and an antibiogram was created using WHONET software.

\section{Results}

From January 2016 to September 2020 a total of 3275 distinct isolates were identified, including 1654 positive blood cultures, 1288 positive urine cultures, and 91 positive cerebrospinal fluid cultures. Aggregate gram negative susceptibility to third generation cephalosporins was approximately $41 \%$, with $67 \%$ of isolates susceptible to piperacillin-tazobactam, and $93 \%$ of isolates susceptible to meropenem. The most frequently identified organism was coagulase-negative Staphylococcus (1534, 47\%), followed by Escherichia coli $(721,22 \%)$, Klebsiella species (482, 15\%), and Staphylococcus aureus (110, 3.4\%). The most common multidrug resistant organism was Escherichia coli $(664,20 \%)$, followed by Klebsiella species $(461,14 \%)$. Acinetobacter baumannii was found to be only $57 \%$ sensitive to meropenem. Staphylococcus aureus was $91 \%$ sensitive to cloxacillin.

\section{Conclusion}

The high rates of antimicrobial resistance found in this rural referral center were similar to the large urban settings in sub-Saharan Africa. This along with the discovery of multidrug resistant gram negative organisms are of great concern. The need for continued surveillance, antimicrobial stewardship, and implementation of quality improvement initiatives is imperative to attempt to curb this burgeoning global problem.

\section{Background}

Antimicrobial resistance (AMR) is a growing threat to healthcare systems worldwide. The World Health Organization (WHO) has declared it one of the top 10 threats to health.(1) There is a large body of evidence and research on the presence and effect of AMR in high-income settings (HIC). However, data from low- and middle-income countries (LMIC) is scarce.(2) Africa and South East Asia were previously identified as regions lacking established AMR surveillance systems.(3) Data that is available has been gathered in large, urban academic hospitals, leaving rural facilities in the dark.(4) Even in the resource 
variable setting of Kenya, surveillance capabilities and information are limited.(5) With sepsis causing one in five deaths globally, hospitals and health systems need to be able to track their bacterial resistance patterns.(6) Antibiograms, although a static measure of drug resistance, can be a useful means to perform local surveillance(7). In this study, our multidisciplinary team created the hospital's first antibiogram to try to understand the prevalence of AMR in a rural setting.

\section{Methods}

Permission to perform this study was granted by the Africa Inland Church (AIC) Kijabe Hospital (KH) Internal Review Board.

\section{Study Setting, Design, and Population}

$\mathrm{KH}$ is a 350-bed rural teaching hospital. Services offered are emergency care, outpatient clinics, general inpatient for adults and children, critical care for adults and children, obstetrics and gynecology, general surgery, and several surgical subspecialties. There are dedicated laboratory and phlebotomy teams that provide most services 24 hours per day, 7 days per week.

This was a retrospective study from blood, urine, and cerebrospinal fluid (CSF) cultures at AIC Kijabe Hospital from February 2016 to September 2020. All specimens collected from either outpatient or inpatient adult and pediatric patients with suspected infections were included. The standard practices at $\mathrm{KH}$ for the preparation of bacterial cultures during the time of the study were as follows.

Blood samples were inoculated in BACTEC bottles and incubated in a BACTEC BD40๑. Following growth detection, the culture media was subsequently placed on an agar plate for further incubation. After colony-forming units (CFUs) were appreciated, gram stains were performed.

Urine or CSF samples were suspended and plated on one of several types of differential media (e.g., MacConkey agar, CLED [cysteine-, lactose-, and electrolyte-deficient] agar, Mannitol agar). Once CFUs were appreciated, gram stains were performed.

From 2016 to 2018, gram negative organisms were further speciated using Analytical Profile Index® (API) kits. To determine sensitivity to antimicrobial agents, the Kirby Bauer disc diffusion method was utilized. Based on Clinical Laboratory and Standards Institute (CLSI) guidelines, zone of growth inhibition was measured to determine whether an isolate was sensitive, intermediate, or resistant to a particular antibiotic agent.(8) Starting in 2018, API kits were no longer used, and speciation and susceptibilities were performed using a Vitek® 2 COMPACT machine.

Gram positive organisms were speciated using stepwise bacterial identification tests (e.g., catalase, oxidase, coagulase tests). Following speciation, sensitivities were obtained using the Kirby Bauer disc diffusion method, as described above. From 2018 on, speciation and susceptibility testing for gram positive organisms were performed using the Vitek ${ }^{\circledR} 2$ COMPACT machine. 
Once speciation and susceptibility testing were completed, the results were either manually entered into a logbook by the laboratory staff or a Vitek ${ }^{\circledR} 2$ COMPACT report was generated. Infection prevention and control staff subsequently transcribed the data into a Microsoft Excel spreadsheet. Using BacLink software, the data from the Excel spreadsheet was then converted into a WHONET compatible format for analysis. Results from 2016-2020 were aggregated to achieve the minimum number of isolates (30) required for analysis.(9) Ten isolates were omitted prior to the analysis due to erroneous data. Per antibiogram standards, only one isolate per patient was utilized in each of the analyses.(9)

\section{Outcome}

The primary outcome was to determine prevalence of antimicrobial resistance in $\mathrm{KH}$ by establishing the hospital's first antibiogram. AMR organisms were defined as those being resistant to one or more classes of antimicrobial agents.(1)

The secondary outcome was the dissemination of bacterial culture information to the hospital at large in a concise format.

\section{Statistical Analysis}

No statistical analyses were performed in this retrospective review.

\section{Results}

From February 2016 to September 2020, 3275 distinct isolates were appreciated. Some isolates were identified in multiple culture samples and were counted only once in determining the total isolate number. There were 1654 positive blood cultures, 1288 positive urine cultures, and 91 positive CSF cultures. Table 1 lists the most common species identified. Of the 3275 distinct isolates, 1534 (46.8\%) were coagulase negative Staphylococcus (CoNS), 721 (22.0\%) were Escherichia coli, 482 (14.7\%) were Klebsiella species, and $110(3.4 \%)$ were Staphylococcus aureus.

Table 1: Most common bacterial isolates at $\mathrm{KH}$ by year 


\begin{tabular}{|lllllll|}
\hline Organism & $\begin{array}{l}\text { Number of isolates } \\
(\%)\end{array}$ & 2016* & $\mathbf{2 0 1 7}$ & $\mathbf{2 0 1 8}$ & $\mathbf{2 0 1 9}$ & $\mathbf{2 0 2 0 +}$ \\
\hline $\begin{array}{l}\text { fStaphylococcus, coagulase } \\
\text { negative }\end{array}$ & $1534(47)$ & 360 & 393 & 471 & 191 & 115 \\
\hline Escherichia coli & $721(22)$ & 101 & 148 & 199 & 203 & 68 \\
\hline Klebsiella pneumoniae & $312(9.5)$ & 59 & 63 & 86 & 66 & 37 \\
\hline Klebsiella oxytoca & $153(4.7)$ & 20 & 45 & 49 & 28 & 11 \\
\hline Staphylococcus aureus & $110(3.4)$ & 36 & 23 & 31 & 12 & 8 \\
\hline Enterobacter cloacae & $63(1.9)$ & 14 & 17 & 15 & 10 & 7 \\
\hline Acinetobacter baumannii & $50(1.5)$ & 8 & 13 & 16 & 9 & 4 \\
\hline Pseudomonas aeruginosa & $39(1.2)$ & 6 & 14 & 11 & 6 & 2 \\
\hline Serratia fonticola & $31(0.9)$ & & 5 & 14 & 6 & 6 \\
\hline
\end{tabular}

*Data from 2016 started in February. TData from 2020 is incomplete (Jan - Sep) due to the burden induced by the COVID-19 pandemic. ¥Not considered pathogenic at $\mathrm{KH}$ outside of neonates or patients with central venous catheterization.

Aggregate susceptibility patterns of gram negative organisms are shown in Figure 1. Collectively, gram negative organisms were found to be less than $50 \%$ susceptible to third and fourth-generation cephalosporins ( $42.0 \%$ ceftazidime, $41.2 \%$ ceftriaxone, $41.7 \%$ cefotaxime, and $47.8 \%$ cefepime), while only $66.5 \%$ susceptible to piperacillin-tazobactam, $86.5 \%$ susceptible to amikacin, and $93 \%$ susceptible to meropenem. Evaluating specific gram negative organisms, Escherichia coli was found to be susceptible to third and fourth generation cephalosporins at higher rates than other gram negative organisms ( $47.8 \%$ ceftazidime, $49.0 \%$ ceftriaxone, $49.3 \%$ cefotaxime, and $51.5 \%$ cefepime), while $75.2 \%$ susceptible to piperacillin-tazobactam, $87.7 \%$ susceptible to amikacin, and $96.1 \%$ susceptible to meropenem. Klebsiella species were less sensitive ( $24.6 \%$ ceftazidime, $26.6 \%$ ceftriaxone, $26.5 \%$ cefotaxime, and $30.3 \%$ cefepime), with $50.5 \%$ susceptible to piperacillin-tazobactam, $88.2 \%$ susceptible to amikacin, and $94.1 \%$ susceptible to meropenem. Acinetobacter baumannii was found to have lower rates of susceptibility for all antibiotics tested (29.2-33.3\% for third generation cephalosporins), $46.3 \%$ susceptible to cefepime, $43.5 \%$ susceptible to piperacillin-tazobactam, $71.9 \%$ susceptible to amikacin, and $57.1 \%$ susceptible to meropenem. In Table 2, the complete susceptibility profile for the most common organisms is listed.

Table 2: AIC Kijabe Hospital Antibiogram 


\begin{tabular}{|c|c|c|c|c|c|c|c|c|c|c|c|c|c|c|c|c|c|c|c|}
\hline $\begin{array}{l}\text { AlC Kijabe } \\
\text { Hospital } \\
\text { Antibiogram, } \\
\text { February } 2016 \\
\text { through } \\
\text { September } \\
2020 \\
\end{array}$ & Organism & 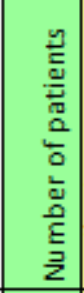 & 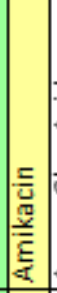 & 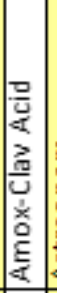 & & & & & : & 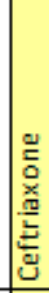 & 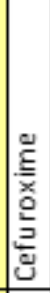 & 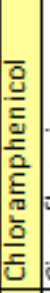 & & 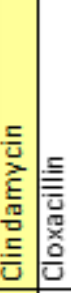 & : & & 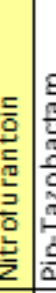 & 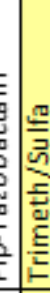 & 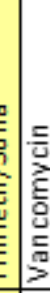 \\
\hline \multirow[t]{7}{*}{ Gram Negative } & Acinetobacter baumannii & 50 & 72 & & & & 46 & & 33 & 29 & & & 51 & & 65 & 57 & 4 & 43. & \\
\hline & Enterobacter cloacae & 63 & 82 & & & & 56 & & 35 & 31 & & 63 & 60 & & 46 & 905 & $54 \mid 6$ & & 5 \\
\hline & Escherichia coli & 721 & 88 & 57. & 49 & & 524 & 498 & 148 & 49 & 47 & 78 & 55 & & & 968 & $85 \mid 7$ & & 9 \\
\hline & Klebsiella oxytoca & 153 & 91 & 38 & 34 & & 35 & & 831 & 133 & 30 & 56 & 60 & & 49 & 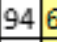 & 605 & $7 \mid 28$ & 8 \\
\hline & Klebsiella pneumoniae & 312 & 87 & 32 & 22 & & 282 & 228. & 522 & 24 & 21 & 62 & 62 & & 35 & $94 \sqrt{4}$ & $4 5 \longdiv { 4 }$ & 7 2.2. & 2 \\
\hline & Pseudomonas aeruginosa & 39 & 82 & & & & 70 & & 6 & & & & 76 & & & 82 & 7 & 4 & \\
\hline & Serratia fonticola & 31 & 97 & 54 & & & 52 & 7 & 5 & 29 & 29 & & 30 & & & 978 & $84 \sqrt{6}$ & & 30 \\
\hline \multirow[t]{2}{*}{ Gram Positive } & Staphylococcus aure us & 110 & & & & 87 & & & & & & 90 & & \begin{tabular}{l|l|l}
66 & 91 \\
\end{tabular} & & & & & \\
\hline & Staphylococcus, coagulase negative & 1534 & & & & 70 & & & & & & 87 & & \begin{tabular}{l|l|l}
57 & 69 \\
\end{tabular} & & & & & \\
\hline
\end{tabular}

This is aggregated culture data from blood, urine, and CSF for the years 2016 to 2020 . The minimum of 30 isolates were used to create the antibiogram. Numbers expressed as percent sensitive.

Regarding gram positive organisms, Staphylococcus aureus was $31.7 \%$ susceptible to trimethoprim/sulfamethoxazole, $65.7 \%$ susceptible to clindamycin, $87.2 \%$ susceptible to cefazolin, and $90.5 \%$ susceptible to cloxacillin. Testing against oxacillin or methicillin was not routinely performed at $\mathrm{KH}$.

Figure 2 details the total percentage of gram negative organisms identified as MDR by year. 2016 was $99.6 \%(259 / 260)$, followed by $88.2 \%$ (301/341) in $2017,94.1 \%(411 / 437)$ in $2018,91.9 \%(351 / 382)$ in 2019 , and 92.3\% (144/156) in 2020 (January through September only). The most frequent gram negative MDR organisms identified at KH were Escherichia coli (664, 20\%) and Klebsiella species (461, 14\%). The total percentage of gram positive organisms identified as MDR in 2016 was $57.9 \%$ (44/76), 77.8\% $(21 / 27)$ in $2017,96.8 \%(30 / 31)$ in $2018,28 \%(9 / 32)$ in 2019 , and $46.2 \%(6 / 13)$ in 2020.

\section{Discussion}

In this study, the multidisciplinary team at $\mathrm{KH}$ set out to establish AMR surveillance with an antibiogram. The creation of an antibiogram helped elucidate the prevalence of AMR in the rural, resource limited setting of $\mathrm{KH}$. This discovery aligns with what has been previously published in large urban settings in East and West Africa.(3)(4)(10)

There was a significant number of CoNS noted in our culture data. At KH this is not considered pathogenic outside of the neonatal period. Another patient population where this organism would be problematic is in individuals with central venous catheters. However, at $\mathrm{KH}$ due to a number of factors, central venous catheters are rarely used. Eliminating CoNS positive blood cultures isolated outside of the neonatal population reduced the total CoNS specimen number to 466 and made the largest percentage of pathogens gram negative organisms. This finding of gram negative organisms being the most common pathogen has been described elsewhere in the region.(3) The largest percentage of MDR organisms were 
also gram negative regardless of specimen source.(3)(11)(12) Although the specific pathogens from these studies vary from those isolated at $\mathrm{KH}$, the overarching theme of MDR gram negative organisms remains.

Ceftriaxone has been used as a broad-spectrum first-line agent at $\mathrm{KH}$ for many years, but the evidence presented by the antibiogram makes it apparent that regimen changes are warranted. Like many institutions in this context, we are limited in our formulary. A next step for $\mathrm{KH}$ is to use piperacillintazobactam, but as the antibiogram has pointed out the most commonly cultured gram negative organism is only $75 \%$ susceptible. This could possibly be overcome by utilizing extended infusions.(13, 14)

Additionally, the percentage of carbapenem resistant (CR) organisms, especially Acinetobacter, is of significant concern. Acinetobacter and Enterobacteriaceae have been identified as critical priority pathogens by the WHO that require special attention in addressing their effect on patients in LMICs.(15) An AMR surveillance and genetic sequencing study from the regional referral center in Nairobi identified 11 different carbapenemase genes in various gram negative pathogens suggesting that the presence of carbapenem resistance is more prevalent in Kenya than previously thought.(15)

Other future directions from this study will be expanding the already formed Infection Prevention and Control team into an Antimicrobial Stewardship team to help improve hospital wide education and uptake of best practices.(16) Additionally, the antibiogram is a static measure of resistance patterns of a particular point in time, and $\mathrm{KH}$ will need to repeat this to continue to monitor these patterns for increasing rates of MDR. Evaluation of MDR trends will have to be viewed in light of the COVID-19 pandemic and the disruption caused to regular hospital functions and admissions. Furthermore, restricting the use of broad-spectrum antimicrobials at $\mathrm{KH}$ will be necessary to thwart selection of more resistant organisms. Not only will a biannual antibiogram be useful in guiding selection of antibiotic agents it will also help identify the areas where quality improvement initiatives can be implemented. For example, moving from identification to determining AMR's impact on patient outcomes will be an important variable to track.

From a regional approach, knowledge translation of our experience to similarly staffed and resourced facilities will be imperative to further grow the local understanding of AMR. Developing collaborative relationships with facilities that are both ahead and behind us in these efforts can expand local knowledge and capacity.

There are several limitations to our study. First, this study was conducted at a single center. Second, there was high laboratory staff turnover during the study period along with changes in the microbiological equipment and availability of materials. This may have led to varying practices in culture preparation, speciation, and ultimately in susceptibility testing. Third, the high rate of CoNS could suggest contamination. Not knowing the total number of cultures obtained over this time eliminate the possibility of determining the contamination rate. Keeping track of this denominator moving forward will allow $\mathrm{KH}$ to compare their rate against accepted standards. Next, the inability to determine which infections are 
community acquired versus hospital acquired limits our ability for quality improvements in these areas. Lastly, the low sensitivity of the WHO MDR definition may have over-selected for the organisms in question. However, in the resource constrained setting, waiting until organisms are resistant to three or more organisms is not a tenable option as there are limited therapeutic choices available.(17)

A major strength of the study is that this is, to our knowledge, a first in a rural hospital, likely representing the AMR currently found in the communities where the majority of Kenyans reside. Additional strengths are the multi-disciplinary team approach, which included laboratory staff, infection control nurses, pharmacy, and several physicians from various departments. Also, this study represents a real-world scenario for many institutions in settings like $\mathrm{KH}$ in that there are many dynamic factors at play. For example, high staff turnover, variability in equipment and reagents, ability to maintain equipment in functional condition, and the collateral effects of a global pandemic are all variables that can affect the consistency of microbiologic data.

\section{Conclusions}

AMR is a global threat that places innumerable lives at risk. Many studies have been conducted to understand the prevalence and impact of AMR on outcomes in HICs, but there is a dearth of objective evidence on AMR from LMIC settings. This study is the first of its kind in looking at the prevalence of AMR in a resource limited hospital in rural Kenya. Similar to HICs and urban centers in LMICs, KH was found to have a large proportion of cultures positive for gram-negative organisms, and many of these that are resistant to commonly used antibiotics. There was also presence of carbapenemase resistant organisms creating therapeutic problems for this resource limited facility. Further quality improvement initiatives, data collection, knowledge translation, and knowledge sharing are needed to address this burgeoning global health problem.

\section{Abbreviations}

AMR - antimicrobial resistance

WHO - World Health Organization

HIC - high income country

LMIC - low income country

AIC - Africa Inland Church

API - Analytical Profile Index

CLSI - Clinical Laboratory and Standards Institute

MDR - multidrug resistance 
$\mathrm{KH}-$ Kijabe Hospital

CSF - cerebrospinal fluid

$\mathrm{CFU}$ - colony forming unit

CoNS - coagulase negative staphylococcus

CR - carbapenem resistance

\section{Declarations}

Ethics approval to perform this retrospective study was obtained by the local IRB at Kijabe Hospital, and a waiver for obtaining consent was granted.

Consent for publication - not applicable as no personal data was included in this manuscript.

Availability of data and materials can be provided by the corresponding author upon request.

Competing interests - The authors have no competing interests.

Funding - none

Author's contributions - GO, AMS, FL, IKB, BJB planned the study, wrote the manuscript, analyzed data; ICD analyzed data with WHONET, wrote the manuscript; JM, VDB, GNW, SKT collected data, proofreading; $\mathrm{KSB}, \mathrm{KL}$ - proofreading and editing of manuscript; STM - data analysis, proofreading and editing of manuscript; El - provided pharmacy expertise, data analysis, proofreading

Acknowledgements - We would like to express our appreciation to all of the patients and their families who were cared for at $\mathrm{KH}$.

We would also like to thank the laboratory staff at $\mathrm{KH}$. Their desire to serve the patients and work tirelessly is inspiring.

\section{References}

1. World Health Organization. Global action plan on antimicrobial resistance. [Internet]. 2015. Available from: http://www.who.int/drugresistance/global_action_plan/en/

2. G. V, C. M, D.M. A, O. D, S. M, Z.A. B. Surveillance for antimicrobial drug resistance in under-resourced countries. Emerg Infect Dis. 2014;20(3):434-41.

3. Tadesse BT, Ashley EA, Ongarello S, Havumaki J, Wijegoonewardena M, González IJ, et al. Antimicrobial resistance in Africa: A systematic review. BMC Infect Dis. 2017;17(1):1-17.

4. Bernabé KJ, Langendorf C, Ford N, Ronat JB, Murphy RA. Antimicrobial resistance in West Africa: a systematic review and meta-analysis. Int J Antimicrob Agents. 2017;50(5):629-39. 
5. Odhiambo F, Boru W, Wences A, Muchemi OM, Kanyina EW, Tonui JC, et al. Antimicrobial resistance: Capacity and practices among clinical laboratories in Kenya, 2013. Pan Afr Med J. 2014;19:1-8.

6. Rudd KE, Johnson SC, Agesa KM, Shackelford KA, Tsoi D, Kievlan DR, et al. Global, regional, and national sepsis incidence and mortality, 1990-2017: analysis for the Global Burden of Disease Study. Lancet. 2020;395(10219):200-11.

7. Truong WR, Hidayat L, Bolaris MA, Nguyen L, Yamaki J. The antibiogram: key considerations for its development and utilization. JAC-Antimicrobial Resist. 2021;3(2):1-6.

8. Performance Standards for Antimicrobial Susceptibility Testing. 28th edition. M100. Clinical And Laboratory Standards Institute. Wayne, PA; 2018.

9. Handler JF, Stelling J. Analysis and presentation of cumulative antibiograms: A new consensus guideline from the Clinical and Laboratory Standards Institute. Clin Infect Dis. 2007;44(6):867-73.

10. Ampaire L, Muhindo A, Orikiriza P, Mwanga-Amumpaire J, Bebell L, Boum Y. A review of antimicrobial resistance in East Africa. Afr J Lab Med. 2016;5(1):1-6.

11. Leopold SJ, van Leth F, Tarekegn H, Schultsz C. Antimicrobial drug resistance among clinically relevant bacterial isolates in sub-Saharan Africa: A systematic review. J Antimicrob Chemother. 2014;69(May):2337-53.

12. Theuretzbacher U. Global antimicrobial resistance in Gram-negative pathogens and clinical need. Curr Opin Microbiol [Internet]. 2015;39(Figure 1):106-12.

13. Rhodes NJ, Liu J, Nicholas O’Donnell J, Dulhunty JM, Abdul-Aziz MH, Berko PY, et al. Prolonged infusion piperacillin-Tazobactam decreases mortality and improves outcomes in severely ill patients: Results of a systematic review and meta-Analysis. Crit Care Med. 2018;46(2):236-43.

14. Chen M, Buurma V, Shah M, Fahim G. Evaluation of studies on extended versus standard infusion of beta-lactam antibiotics. Am J Heal Pharm. 2019;76(18):1383-94.

15. Prioritization of pathogens to guide discovery, research and development of new antibiotics for drugresistant bacterial infections, including tuberculosis. Geneva; http://apps.who.int/iris

16. Tomczyk S, Storr J, Kilpatrick C, Allegranzi B. Infection prevention and control (IPC) implementation in low-resource settings: a qualitative analysis. Antimicrob Resist Infect Control. 2021;10(1):1-11.

17. Magiorakos A, Srinivasan A, Carey R, Carmeli Y, Falagas M, Giske C, et al. Multidrug-resistant, extensively drug-resistant and pandrug-resistant bacteria: an international expert proposal for interim standard definitions for acquired resistance. Clin Microbiol Infect Dis. 2011;18(3):268-81.

\section{Figures}




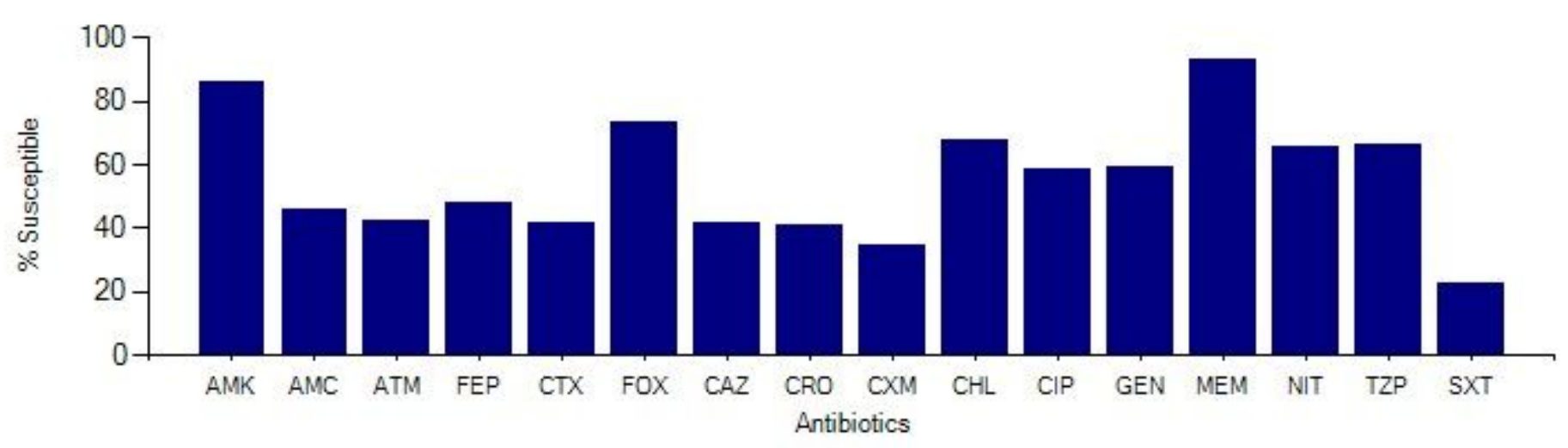

\section{Figure 1}

\section{Aggregate Susceptibility of Gram-Negative Organisms}

All blood, urine, and CSF samples with GNR isolates identified were included and analyzed in aggregate.

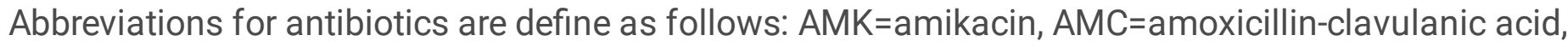
ATM=aztreonam, FEP=cefepime, $C T X=$ cefotaxime, $F O X=$ cefoxitin, $C A Z=$ ceftazidime, $C R O=$ ceftriaxone, $\mathrm{CXM}=$ cefuroxime, $\mathrm{CHL}=$ chloramphenicol, $\mathrm{CIP}=$ ciprofloxacin, $\mathrm{GEN}=$ gentamicin, $\mathrm{MEM}=$ meropenem, $\mathrm{NIT}=$ nitrofurantoin, $\mathrm{TZP}=$ piperacillin-tazobactam, $\mathrm{SXT}=$ trimethoprim/sulfamethoxazole.

\begin{tabular}{|c|c|c|c|c|c|}
\hline $100.00 \%$ & $99.60 \%$ & & & & \\
\hline & & $88.20 \%$ & $94.10 \%$ & $91.90 \%$ & $92.30 \%$ \\
\hline $80.00 \%$ & & & & & \\
\hline $70.00 \%$ & & & & & \\
\hline $60.00 \%$ & & & & & \\
\hline $50.00 \%$ & & & & & \\
\hline $40.00 \%$ & & & & & \\
\hline $30.00 \%$ & & & & & \\
\hline $20.00 \%$ & & & & & \\
\hline $10.00 \%$ & & & & & \\
\hline \multirow{2}{*}{$0.00 \%$} & 2016 & 2017 & 2018 & 2019 & 2020 \\
\hline & & & YEAR & & \\
\hline
\end{tabular}

Figure 2 
Percent of Gram-Negative MDR Organisms by year

All blood, urine, and CSF samples with GNR isolates identified were included. Those found to be MDR were divided by the total number of GNR isolates to obtain the percentages. 